\section{Interview with Dr. Nicola Luigi Bragazzi}

\section{Paraskevi Theofilou}

Sotiria Hospital for Thoracic Diseases, Athens, Greece

Dr. Nicola Luigi Bragazzi is currently resident in Public Health at the National University of Genoa, Faculty of Medicine and Surgery, Italy. His research covers different areas, including: i) psychosocial processes and patient-related outcomes, such as quality of life and health locus of control in chronic illness with a particular emphasis on chronic kidney diseases and multiple sclerosis, ii) self-management and empowerment interventions for patients with chronic illness, and iii) conceptual frameworks for integrating psychology and medicine. His work related to clinical psychology has been published in journals such as the American Journal of Bioethics, Patient Preference and Adherence, Psychology research and behavior management and Multiple Sclerosis International.

\section{Question n. 1}

Your work in the past years has made significant contributions to the literature in health psychology covering a variety of topics. What would you say are the common threads within your research, the core questions you have been preoccupied with?

\section{Answer}

I fully believe in the unity of the sciences and of knowledge: specialized branches are different facets of the same medal. Nowadays, there is an urgent need for an interdisciplinary approach, combining different techniques and points of view. The prominent biologist and founder of sociobiology Edmund Wilson defined this concept as Consilience, ${ }^{1}$ while the physicist and historian Gerald Holton talked of a Ionian Enchantmen, describing the efforts to unite and aggregate, rather than divide and separate. This dream of unified learning (Edmund Wilson) means that, after dissecting and obtaining a wealth of details, one has to address and envisage the inner unity of the complex phenomenon, recovering a global of view of it. Having details is undoubtedly an important step, but it is only the first step towards knowledge. This advocates a holistic approach beyond the reductionism. If you look back at the history of the initial and primeval knowledge, you will find the philosophers (literally in Greek, the word philosophy means love for the knowledge) who dealt with every aspect of life, from biology to astronomy and law. Later, this unitary approach split into two cultures (Charles Percy Snow), ${ }^{2}$ without any communication between them. Robert Costanza has well conveyed the necessity of integrating humanities and the hard sciences, finding a balance between the rigor, the analysis and the synthesis. ${ }^{3}$ Specifically, in the field of psychology and psychoanalysis, some scholars have attempted to follow this, such as the psychoanalyst David Forrest, ${ }^{4}$ and the behavioral psychologist David Wilson. 5

\section{Question n. 2}

Your research interests are related to quality of life, spirituality, health locus of control in different chronic diseases like end-stage renal disease (ESRD) and multiple sclerosis (MS). On the other side, your publications cover a different scientific area which is Biophysics and Nanobiotechnology. How can you combine the above topics?

\section{Answer}

As I told before, I am strongly convinced that endeavors should be undertaken in order to merge and make converge the different sciences and approaches. On the other hand, some unified approaches are already currently available such as neurophysics (namely, the hyper-specialized branch of biophysics studying the functioning of the nervous system) which tries to combine a vast arrays of perspectives, ranging from computational to psycho-cognitive neuroscience. Nanoethics studies the ethical and philosophical implications of the small world and of the industrial applications of the nanobiotechnologies. Other examples are neurorobotics, neuroethics, neuroeconomics, which have considerably progressed by taking into account the last discoveries and findings in the field of psychology.

\section{Question n.3}

One of your papers that you have published focuses on P6 model in clinical medicine and the fact that in the last decades there has been a shift from $\mathrm{P} 0$ medicine to a $\mathrm{P} 6$ model. Could you please give us more details about this model?

\section{Answer}

P6 model is a conceptual framework describing how medicine has become preventive, participatory, predictive, personalized, psychocognitive and public. It has shifted from paternalism to a new patient-physician relation-
Correspondence: Paraskevi Theofilou, Sotiria Hospital for Thoracic Diseases Athens, Greece. Tel. +30.697.744.1502, +30.210 .622 .1435 .

Fax +30.210 .622 .1435$

E-mail: theofi@otenet.gr, paraskevi.theofilou@ gmail.com

Received for publication: 17 June 2014. Accepted for publication: 17 June 2014.

This work is licensed under a Creative Commons Attribution NonCommercial 3.0 License (CC BYNC 3.0).

(c) Copyright P. Theofilou., 2015

Licensee PAGEPress, Italy

Health Psychology Research 2015; 3:1751

doi:10.4081/hpr.2015.1751

ship. Reflecting upon the case-story of Salvatore Iaconesi, who decided to make an open self-disclosure of his recently diagnosed brain tumor, I have shown how the usage of (new) technologies, as well as the advancement in the medical knowledge have shaped a new form of narrative, interactive, and augmented medicine. ${ }^{6,7}$

\section{Question n. 4}

Which are the projects you are working on at the moment in terms of research and/or publications?

\section{Answer}

Currently, I am working on some projects of music psychology in collaboration with engineers and musicians. I am also working on a project of traffic psychology, involving also computer scientists. I like working in multidisciplinary teams.

\section{Question n. 5}

How do you see the relationship between theory and practice for a health psychologist as well as in your own work?

\section{Answer}

I think theory is fundamental for properly addressing several issues, but must be always tested and challenged in real life. Theory needs practice, as well as viceversa. A great philosopher, Immanuel Kant, used to say that the general rule must be supplemented by an act of judgment whereby the practitioner distinguishes instances where the rule applies from those where it does not. ${ }^{8}$ 
is/are the main contribution(s) it made or has to make in the coming future to strengthen its role as one of the most dynamic and important fields of scientific inquiry?

Who are the science personalities whose masterpieces most deeply influenced your professional life?

\section{Answer}

Besides Edmund Wilson, Gerald Holton and Charles Percy Snow, which I already mentioned before, I would like to quote Richard Dawkins, who first introduced the concept of meme, Edmund Pellegrino, the great advocate of the medical humanities, and Susan Sontag who has unraveled the metaphors of diseases, such as cancer and AIDS. Moreover, I have been fascinated by the collaboration between the psychologist Jung and the quantum physicist Pauli. ${ }^{9}$

\section{Question n. 7}

Finally, what do you think is the role of psychology in the contemporary world? What

\section{Answer}

I am impressed by the increasingly expansion of psychology. I think that, in the field of clinical medicine, concepts like quality of life (QOL, HRQOL), health locus of control (HLOC), self-esteem, self-competence, selfmanagement and other patient-related outcomes (PROs) are of fundamental importance. Medicine cannot ignore psychology if wants to attain a better clinical outcome for the patient.

\section{References}

1. Wilson E0. Consilience: the unity of knowledge. New York: Knopf; 1998.

2. Snow CP. The two cultures. London: Cambridge University Press; 1959.

3. Costanza R. A vision of the future of science: reintegrating the study of humans and the rest of nature. Futures 2003;35:651-71.

4. Forrest DV. Edward 0. Wilson's consilience: can our knowledge be unified? J Am Acad Psychoanal 1999;27:371-86.

5. Wilson DS. Consilience: making contextual behavioral science part of the United Ivory archipelago. J Context Behav Sci 2012;1-2:39-42.

6. Bragazzi NL. Children, adolescents, and young adults participatory medicine: involving them in the health care process as a strategy for facing the infertility issue. Am J Bioeth 2013;13:43-4.

7. Bragazzi NL. From P0 to P6 medicine, a model of highly participatory, narrative, interactive, and "augmented" medicine: some considerations on Salvatore Iaconesi's clinical story. Patient Prefer Adherence 2013;7:353-9.

8. Kant I. Kant's political writings. Reiss H, ed. Cambridge: Cambridge University Press; 1970.

9. Jung CG, Wolfgang Pauli W. Naturerklärung und Psyche. Zürich: Rascher Verlag; 1952. 